Arrivoc

Free to Authors and Readers
A Platinum Open Access Journal for Organic Chemistry
Paper

Arkivoc 2021, part viii, 96-106

\title{
L-Ascorbic acid as an efficient organocatalyst for the synthesis of dispiro[tetrahydroquinoline-bis(1,3-dioxane-4,6-dione)] derivatives
}

Peixin Rui, ${ }^{a}$ Zhaohui $X u,{ }^{a *}$ Jingli Liu, ${ }^{b *}$ and Qingshui Huang ${ }^{c *}$

${ }^{a}$ Key Laboratory of Functional Small Organic Molecules, Ministry of Education and College of Chemistry \& Chemical Engineering, Jiangxi Normal University, Nanchang, Jiangxi 330022, China

${ }^{b}$ NanChang Hongdu Hospital of TCM, Nanchang 330006, China

${ }^{c}$ Clinical Laboratory, First Affiliated Hospital of Nanchang University, Nanchang 330006, China

Email: 002776@jxnu.edu.cn; gotoxzhhqs@163.com

Received 01-04-2021

\section{Abstract}

An efficient four-component reaction between aromatic aldehydes, 1,3-dioxane-4,6-dione, arylamines and acetone for the synthesis of dispiro[tetrahydroquinoline-bis(1,3-dioxane-4,6-dione)] derivatives in the presence of L-ascorbic acid as an efficient organocatalyst, is described. The remarkable advantages offered by this protocol are an inexpensive catalyst, good yields, a broad substrate scope, mild conditions and, a simple and easy work-up procedure. This method affords the end products through a combination of the Knoevenagel, Michael, Diels-Alder and an intramolecular reaction.

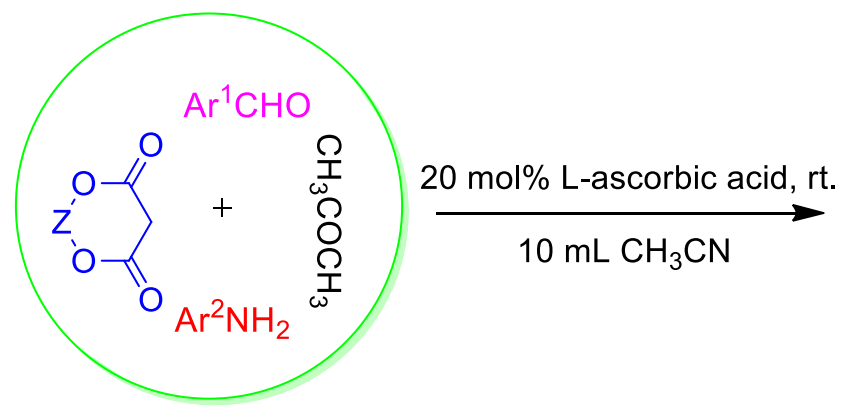

Keywords: dispiro[tetrahydroquinoline-bis(1,3-dioxane-4,6-dione)]derivatives, four-component reaction, Lascorbic acid, 1,3-dioxane-4,6-dione 


\section{Introduction}

Hydroquinoline ring is an important structural feature in many pharmaceutical agents and drug molecules. ${ }^{1-3}$ Some hydroquinoline structures are potent tetrahydroquinoline-derived antibiotics extracted from Janibecter limosus. ${ }^{4}$ Particularly, tetrahydroquinoline derivatives have exhibited a wide range of interesting biological activitives, for example, antiproliferation, ${ }^{5}$ antifungal, ${ }^{6}$ antihyperalgesia, ${ }^{7}$ anticancer, ${ }^{8,9}$ antituberculosis, ${ }^{10}$ neuronal nitric oxide synthase (nNOS) inhibition, ${ }^{11} 5-\mathrm{HT}_{2} \mathrm{C}$ receptor agonism, ${ }^{12}$ anti-Inflammatory, ${ }^{13}$ and antiHIV activity. ${ }^{14}$ Tetrahydroquinolines including Meldrum's acid structure can provide attractive building blocks for the synthesis of natural products. They are also the starting materials for the synthesis of exotic amino acids that are used to modify the physical properties and biological activities of peptides, peptidomimetics, and proteins. ${ }^{15,16}$ Therefore, the development of new methods for the synthesis of hydroquinoline ring compounds is of continuous interest to synthetic as well as medicinal chemists.

Multicomponent reactions with features such as easy operation, high efficiency, high selectivity and atomic economy are considered to be the closest to the ideal synthesis process and have been widely used in the fields of the total synthesis of natural products, heterocyclic compounds and combinatorial chemistry. ${ }^{17,18}$ Diastereoselective synthesis of dispiro[tetrahydroquinoline-bis(2,2-dimethyl[1,3]dioxan-4,6-dione)] derivatives through a three-component reaction of amines, aromatic aldehydes, and Meldrum's acid has been reported in the presence of various Brønsted acids such as acetic acid, ${ }^{19}$ benzoic acid, ${ }^{20}$ trichloroacetic acid, ${ }^{21}$ citric acid, ${ }^{22}$ salicylic acid, ${ }^{23}$ phthalic acid. ${ }^{24}$ 4-(sulfobutyl)tris(4-sulfophenyl)phosphonium hydrogen sulfate can also achieve good catalytic effect in this reaction ${ }^{25}$ (Scheme 1). However, many of these methodologies are associated with limitations such as low yields, long reaction times, high catalyst loading, and environmentally unfavorable solvents. Therefore, an efficient, and enviromentally friendly method for the synthesis of hydroquinoline compounds is certainly desirable.

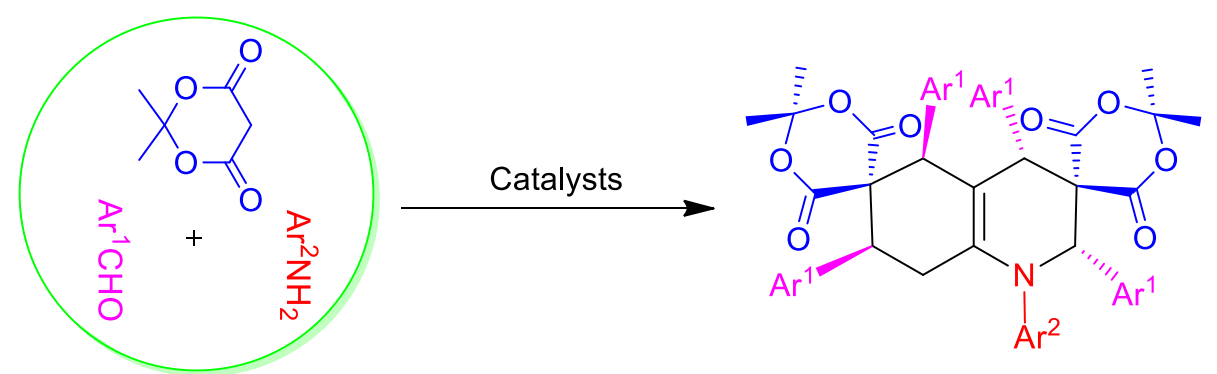

Catalysts: Acetic acid, benzoic acid, trichloroacetic acid, citric acid, salicylic acid, phthalic acid, 4-(sulfobutyl)tris(4-sulfophenyl)phosphonium hydrogen sulfate

Scheme 1. Reported synthesis of dispiro[tetrahydroquinoline-bis(2,2-dimethyl-1,3-dioxane-4,6-dione)] derivatives.

Ascorbic acid, also known as vitamin C, is a polyhydroxy compound with acidic and reducing properties. In recent years, it has received considerable attention as a green, mild and inexpensive catalyst for various organic transformations, such as multicomponent reactions, ${ }^{18,26,27}$ the degradation of cyanidin-3-O- $\beta$ glucoside, ${ }^{28}$ benzaldehyde formation, ${ }^{29}$ and ipso-hydroxylation of arylboronic acid. ${ }^{30}$ Due to its wide applicability as an efficient organocatalyst, L-ascorbic acid is herein reported as an effective catalyst for the synthesis of dispiro[tetrahydroquinoline-bis(1,3-dioxane-4,6-dione)] derivatives via a four-component reaction involving an initial Knoevenagel reaction, followed by a Diels-Alder, Michael addition and finally an intramolecular reaction (Scheme 2). 


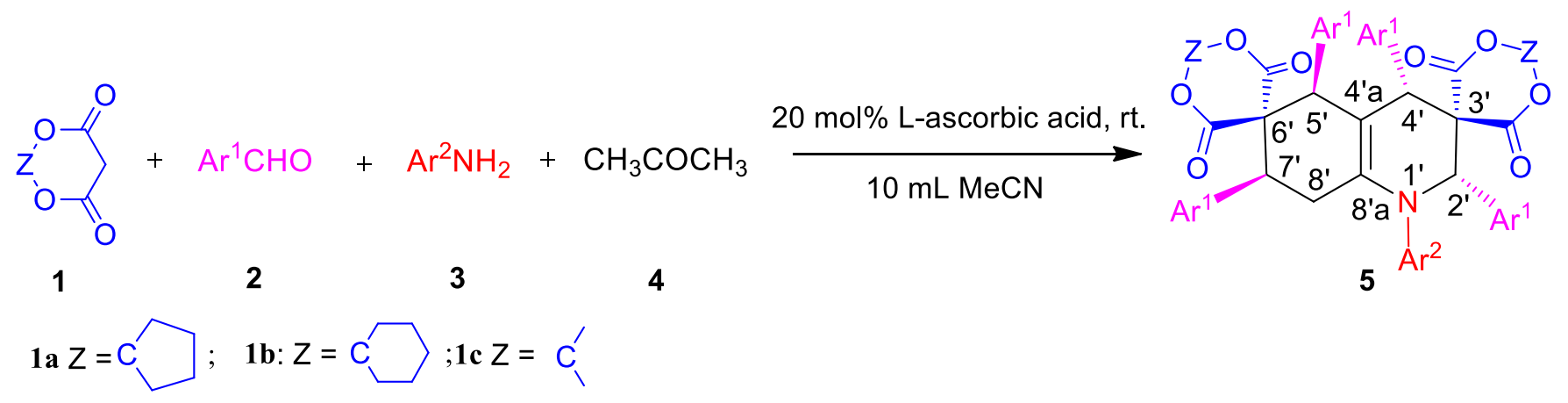

Scheme 2. The new four-component synthesis of dispiro[tetrahydroquinoline-bis(1,3-dioxane-4,6-dione)] derivatives.

\section{Results and Discussion}

To obtain the optimized reaction conditions, the reaction of 2,2-butylidene-1,3-dioxane-4,6-dione (1a), benzaldehyde (2a), aniline (3a) and acetone (4) was chosen as a model reaction. In our initial screening experiments, the effects of solvents were investigated. Different solvents including water, ethanol, methanol, acetone and acetonitrile were used without any catalyst (Table 1, entries 1-5). Results show that the yield only reached $37 \%$ in $\mathrm{CH}_{3} \mathrm{CN}$ under catalyst-free conditions (Table 1, entry 5). After screening different catalysts such as $\mathrm{I}_{2}$, $\mathrm{H}_{3} \mathrm{PO}_{3}$, a gluconic acid aqueous solution (50 wt\%, GAAS), Phthalimide and ascorbic acid, it was found that the best yield was obtained in the presence of 20 mol\% L-ascorbic acid in $\mathrm{CH}_{3} \mathrm{CN}$ (Table 1, entries 6-10). Encouraged by these lead results, the model reaction was performed at different concentrations of malic acid resulting in different yields Finally, we determined 20 mol\% L-ascorbic acid was sufficient to push the reaction to completion. Higher amounts of the catalyst did not improve the results to any greater extent. Optimal results for the reaction conditions of 2,2-butylidene-1,3-dioxane-4,6-dione (1a, 2 mmol), benzaldehyde (2a, 4 $\mathrm{mmol}$ ), aniline (3a, $1 \mathrm{mmol}$ ) and acetone $(4,2 \mathrm{mmol})$ as reactant in $\mathrm{CH}_{3} \mathrm{CN}$ furnished $5 \mathrm{a}$ in $82 \%$ yield.

Table 1. Optimization of reaction conditions for the synthesis of $\mathbf{5} \mathbf{a}^{\mathbf{a}}$

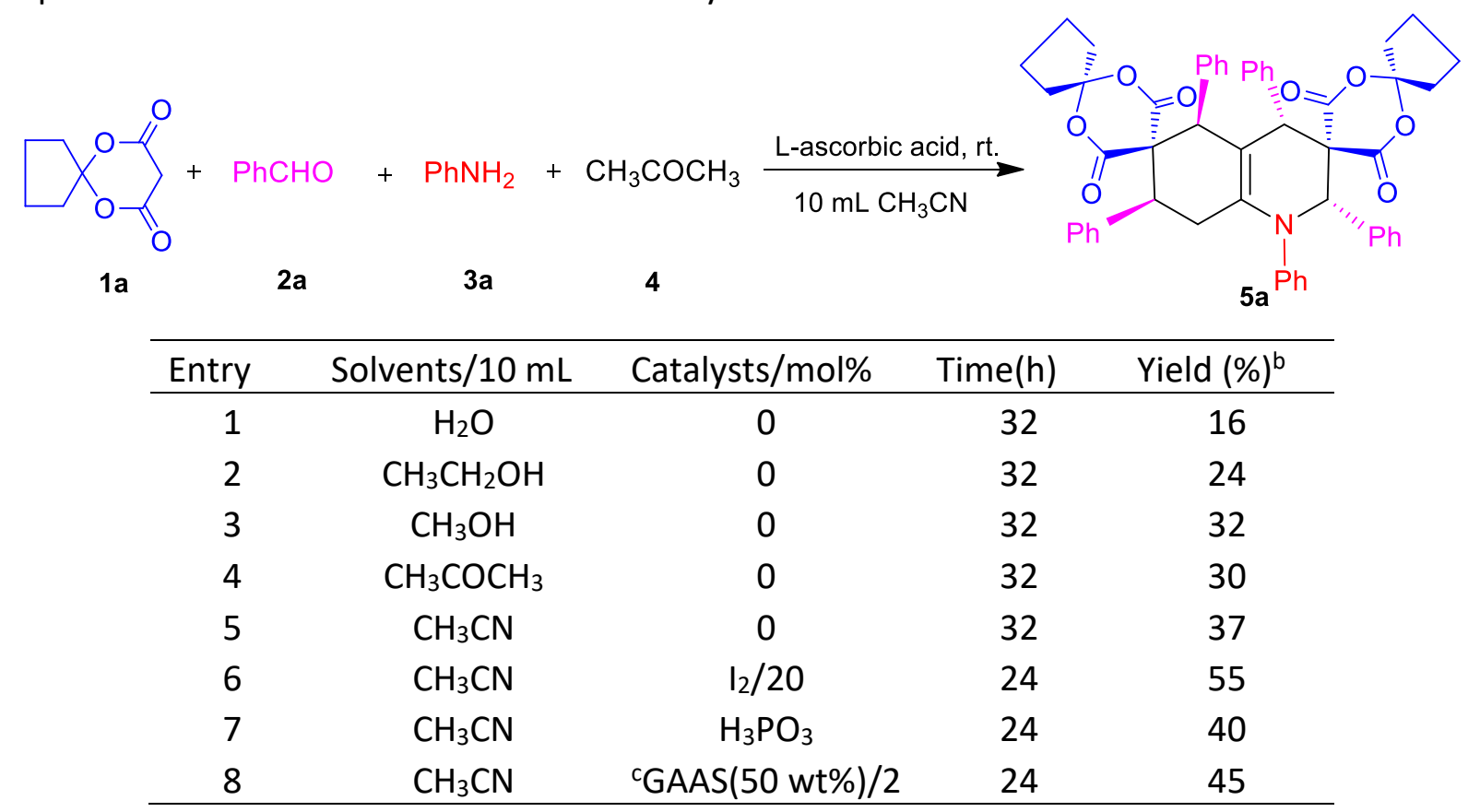


Table 1. Continued

\begin{tabular}{ccccc}
\hline Entry & Solvents/10 mL & Catalysts/mol\% & Time(h) & Yield (\%) $^{\text {b }}$ \\
\hline 9 & $\mathrm{CH}_{3} \mathrm{CN}$ & Phthalimide/20 & 24 & 48 \\
10 & $\mathrm{CH}_{3} \mathrm{CN}$ & Ascorbic acid/20 & 20 & 82 \\
11 & $\mathrm{CH}_{3} \mathrm{CN}$ & Ascorbic acid/15 & 30 & 75 \\
12 & $\mathrm{CH}_{3} \mathrm{CN}$ & Ascorbic acid/25 & 24 & 80 \\
\hline
\end{tabular}

a Reaction conditions: 2,2-butylidene-1,3-dioxane-4,6-dione (1a, $2 \mathrm{mmol}$ ), benzaldehyde (2a, $4 \mathrm{mmol})$, aniline $(3 \mathrm{a}, 1 \mathrm{mmol})$ and acetone $(4,2 \mathrm{mmol})$ at room temperature; ${ }^{b}$ Isolated yield; ${ }^{c}$ Gluconic acid aqueous solution (GAAS).

Under optimized conditions, a number of substrates including different aldehydes and arylamines have been investigated. Aromatic aldehydes bearing either electron-withdrawing functional groups, such as $\mathrm{F}$ and $\mathrm{Cl}$ substituents, or electron-donating groups, such as $\mathrm{CH}_{3}$ and $\mathrm{CH}_{3} \mathrm{O}$, are tolerated leading to good yields. Good results were also obtained using arylamines with either electron-withdrawing, such as $\mathrm{F}$, or electron-donating groups such as $\mathrm{CH}_{3}$ and $\mathrm{CH}_{3} \mathrm{O}$ substituents. 2,2-Pentylidene-1,3-dioxane-4,6-dione (1b) and 2,2-dimethyl-1,3dioxane-4,6-dione (1c) could also be used to produce $\mathbf{5 i - 5 j}$ in high yields. All the results are summarized in Table 2.

Table 2. Synthesis of dispiro[tetrahydroquinoline-bis(1,3-dioxane-4,6-dione)] derivatives ${ }^{a}$<smiles>[Z]O[C@H]1CC(=O)O[C]O1</smiles>

1
$+\mathrm{Ar}^{1} \mathrm{CHO}+\mathrm{Ar}^{2} \mathrm{NH}_{2}+\mathrm{CH}_{3} \mathrm{COCH}_{3}$ O

$2 a-2 e$

$3 a-3 d$

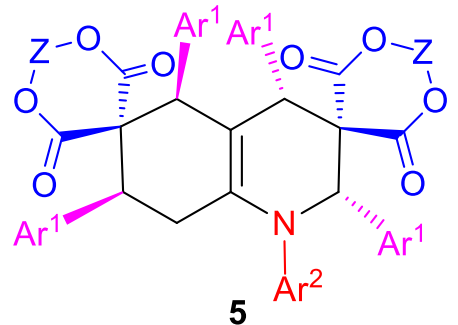

20 mol\% L-ascorbic acid, rt. $10 \mathrm{~mL} \mathrm{CH} \mathrm{CN}_{3}$

4

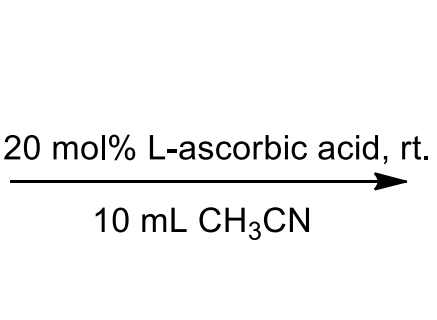

5<smiles>[Y18]C(=[Z10])C1CCCC1</smiles>

\begin{tabular}{ccccccc}
\hline Entry & Compounds 1 & $\mathrm{Ar}^{1}$ & $\mathrm{Ar}^{2}$ & Time(h) & Product & Yields (\%) \\
\hline 1 & $\mathbf{1 a}$ & $\mathbf{2 a}\left(\mathrm{C}_{6} \mathrm{H}_{5}\right)$ & $\mathbf{3 a}\left(\mathrm{C}_{6} \mathrm{H}_{5}\right)$ & 20 & $\mathbf{5 a}$ & 82 \\
2 & $\mathbf{1 a}$ & $\mathbf{2 b}\left(4-\mathrm{FC}_{6} \mathrm{H}_{4}\right)$ & $\mathbf{3 a}\left(\mathrm{C}_{6} \mathrm{H}_{5}\right)$ & 15 & $\mathbf{5 b}$ & $\mathbf{7 4}$ \\
3 & $\mathbf{1 a}$ & $\mathbf{2 c}\left(4-\mathrm{ClC}_{6} \mathrm{H}_{4}\right)$ & $\mathbf{3 a}\left(\mathrm{C}_{6} \mathrm{H}_{5}\right)$ & 16 & $\mathbf{5 c}$ & $\mathbf{7 7}$ \\
4 & $\mathbf{1 a}$ & $\mathbf{2} \mathbf{d}\left(4-\mathrm{CH}_{3} \mathrm{C}_{6} \mathrm{H}_{4}\right)$ & $\mathbf{3 a}\left(\mathrm{C}_{6} \mathrm{H}_{5}\right)$ & 24 & $\mathbf{5 d}$ & 65 \\
5 & $\mathbf{1 a}$ & $\mathbf{2 e}\left(4-\mathrm{CH}_{3} \mathrm{OC}_{6} \mathrm{H}_{4}\right)$ & $\mathbf{3 a}\left(\mathrm{C}_{6} \mathrm{H}_{5}\right)$ & 24 & $\mathbf{5 e}$ & 63 \\
6 & $\mathbf{1 a}$ & $\mathbf{2 a}\left(\mathrm{C}_{6} \mathrm{H}_{5}\right)$ & $\mathbf{3 b}\left(4-\mathrm{FC}_{6} \mathrm{H}_{4}\right)$ & 24 & $\mathbf{5 f}$ & $\mathbf{7 2}$ \\
7 & $\mathbf{1 a}$ & $\mathbf{2 a}\left(\mathrm{C}_{6} \mathrm{H}_{4}\right)$ & $\mathbf{3 c}\left(4-\mathrm{CH}_{3} \mathrm{OC}_{6} \mathrm{H}_{4}\right)$ & 30 & $\mathbf{5 g}$ & 70 \\
8 & $\mathbf{1 a}$ & $\mathbf{2 a}\left(\mathrm{C}_{6} \mathrm{H}_{4}\right)$ & $\mathbf{3 d}\left(4-\mathrm{CH}_{3} \mathrm{C}_{6} \mathrm{H}_{4}\right)$ & 20 & $\mathbf{5 h}$ & $\mathbf{7 6}$ \\
9 & $\mathbf{1 b}$ & $\mathbf{2 a}\left(\mathrm{C}_{6} \mathrm{H}_{5}\right)$ & $\mathbf{3 a}\left(\mathrm{C}_{6} \mathrm{H}_{5}\right)$ & 20 & $\mathbf{5 i}$ & 82 \\
10 & $\mathbf{1 c}$ & $\mathbf{2 a}\left(\mathrm{C}_{6} \mathrm{H}_{5}\right)$ & $\mathbf{3 a}\left(\mathrm{C}_{6} \mathrm{H}_{5}\right)$ & 18 & $\mathbf{5 j}$ & 85 \\
\hline
\end{tabular}

aReaction conditions: 1,3-dioxane-4,6-dione (1a, $2 \mathrm{mmol})$, different aldehydes(2, $4 \mathrm{mmol})$, arylamines(3, $1 \mathrm{mmol})$, acetone(4, $2 \mathrm{~mol}), 20 \mathrm{~mol} \% \mathrm{~L}$-ascorbic acid in $10 \mathrm{~mL} \mathrm{CH}_{3} \mathrm{CN}$ at room temperature; ${ }^{b}$ Isolated yield. 
The structure of $1^{\prime}, 2^{\prime}, 4^{\prime}, 5^{\prime}, 7^{\prime}$-pentaphenyl-1'H-dispiro[1',5',7',8'-tetrahydroquinoline-5,3':6',5'-bis(2,2pentylidene-1,3-dioxane-4,6-dione)] (5i) was also established by X-ray crystallography (Figure 1) and by NMR spectroscopy.

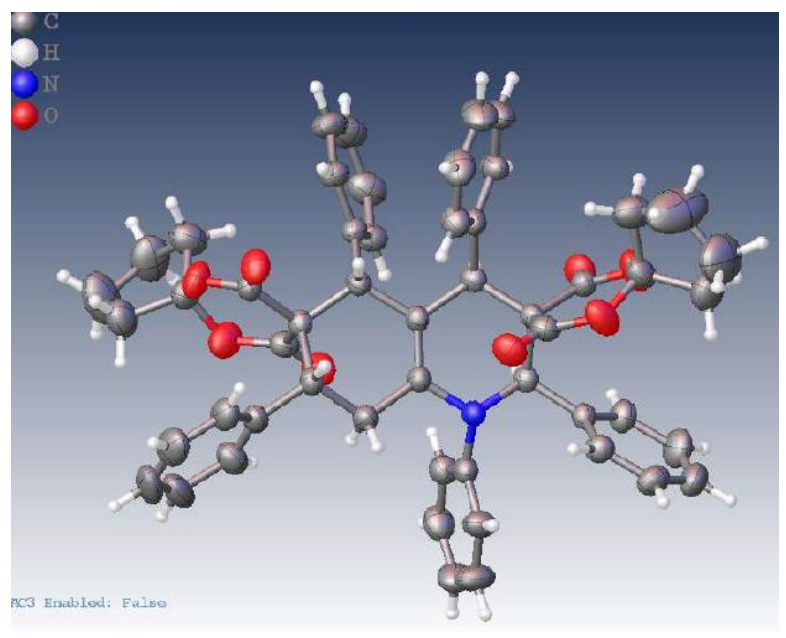

Figure 1. X-ray crystal structure of $\mathbf{5 i .}$

A plausible mechanism for the synthesis of $1^{\prime}, 2^{\prime}, 4^{\prime}, 5^{\prime}, 7^{\prime}$-pentaphenyl-1' $H$-dispiro[ $\left[1^{\prime}, 5^{\prime}, 7^{\prime}, 8^{\prime}-\right.$ tetrahydroquinoline-5,3':6',5'-bis(2,2-butylidene-1,3-dioxane-4,6-dione)] 5a is depicted in Scheme 3. The product 5a was obtained through the combination of a Knoevenagel condensation, a Diels-Alder, a Michael addition and an intramolecular reaction. ${ }^{24}$ For the formation of benzylidene Meldrum's acid 6, ${ }^{31} \mathrm{~L}$-ascorbic acid promoted the enolization of $1 \mathrm{a}$ by forming hydrogen bonds with the $-\mathrm{OH}$ of $1 \mathrm{a}$, thus increasing the nucleophilic character of the methylene carbon of 1a. Meanwhile, it also increases the electrophilic character of the carbonyl of $\mathbf{2 a}$ by forming hydrogen bonds with the carbonyl oxygen of $\mathbf{2 a}$. After the condensation reaction, benzylidene Meldrum's acid 6 is obtained. For the formation of 2-amino-1,3-butadiene 10, acetone undergoes a condensation with aniline to give imine $\mathbf{7}$ which tautomerizes to enamine $\mathbf{8}$. Compound $\mathbf{8}$ then reacts with phenyl aldehyde to form the reactive 2-amino-1,3-butadiene 10 . Further to form product 5a, 2amino-1,3-butadiene 10 acts as an activated 1,3-diene, and a concerted [4 +2] cycloaddition can take place with benzylidene Meldrum's acid 6 as dienophile (Diels-Alder) to furnish enamine 11. enamine 11 with benzylidene Meldrum's acid generates 12 by Michael addition, then forming Compound 13 with phenyl aldehyde by addition reaction, which then undergoes intramolecular reaction leading to $\mathbf{5 a}$. 
<smiles>O=C1OC2(CCCC2)OC(=O)C1COCCO</smiles>

$1 \mathrm{a}$<smiles>C[C@@H]1OC(=O)[C@@]2(C)CCC1(O)COC2=O</smiles>

2a

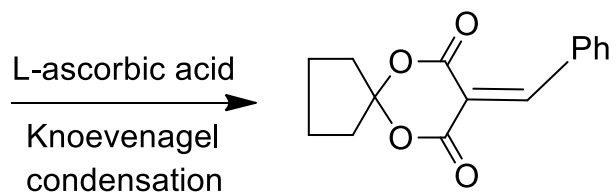

6

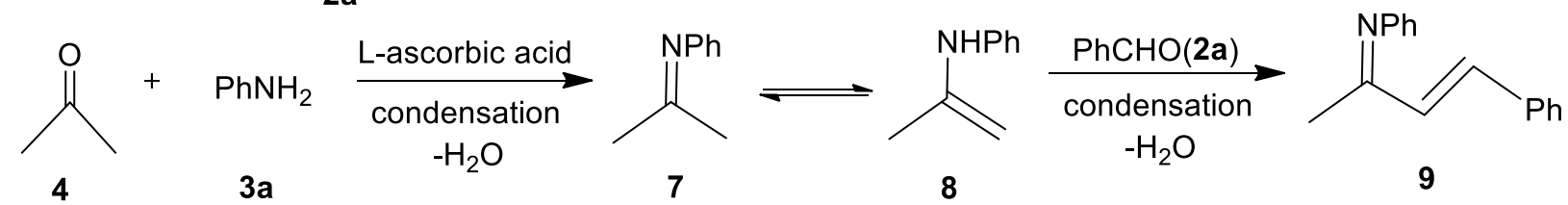

4

$3 a$

7

8

9<smiles>C=CC(C=Cc1ccccc1)Nc1ccccc1</smiles>

10<smiles>C=C1C(=O)OC2(CCCC2)OC1=O</smiles>

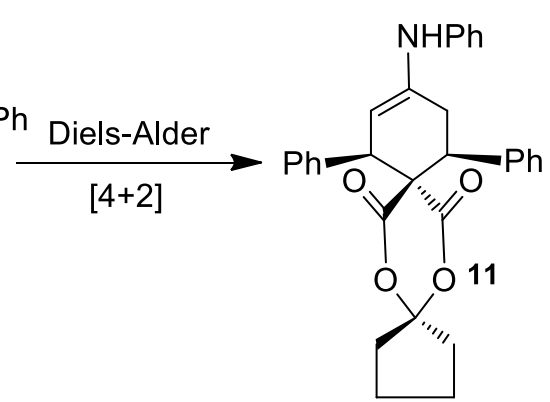<smiles>O=C1OC2(CCCC2)OC(=O)C1=Cc1ccccc1</smiles><smiles>NC1=C([C@H](c2ccccc2)C2C(=O)OC3(CCCC3)OC2=O)[C@@](C(=O)O)(c2ccccc2)[C@H](Pc2ccccc2)C1</smiles>

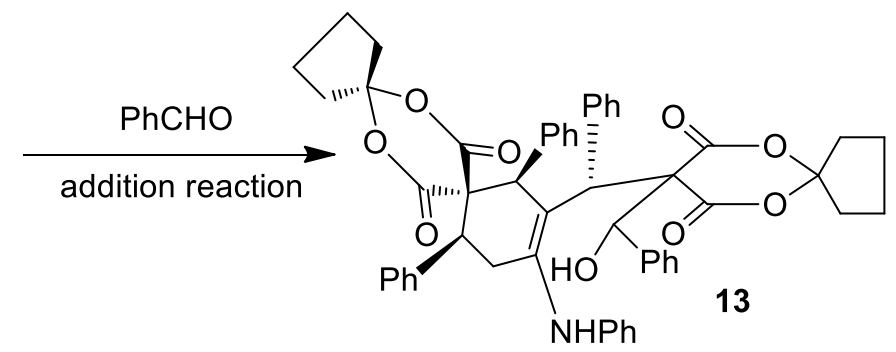

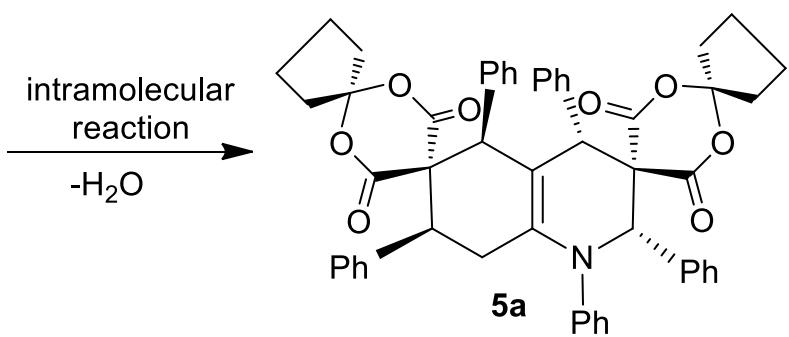

Scheme 3. Proposed mechanism for the formation of $\mathbf{5 a .}$

\section{Conclusions}

In conclusion, an efficient four-component reaction between aromatic aldehydes, 1,3-dioxane-4,6-dione, arylamines and acetone for the synthesis of dispiro[tetrahydroquinoline-bis(1,3-dioxane-4,6-dione)] derivatives in good yields was reported catalyzed by L-ascorbic acid. The operation and work-up procedures 
are straightforward and no column chrommatography purification is needed as well. These advantages will make this method a valid contribution to existing methodologies for hydroquinoline containing ring compounds.

\section{Experimental Section}

General. 1,3-dioxane-4,6-dione was prepared according to literature. ${ }^{32,33}$ The other chemicals were purchased from Aladdin, Aldrich and Fluka Chemical Companies and without further purification. Melting points were measured on XT-4 digital micro melting point apparatus and are uncorrected. ${ }^{1} \mathrm{H}$ NMR spectra were recorded on a BRUKER AVANCE $400 \mathrm{MHz}$ spectrometer using $\mathrm{CDCl}_{3}$ as the solvent and TMS as the internal standard. ${ }^{13} \mathrm{C}$ NMR data were collected on a BRUKER AVANCE $100 \mathrm{MHz}$ instrument with $\mathrm{CDCl}_{3}$ as the solvent and TMS as the internal standard. The analytical MS of the compounds was performed on Agilent LC-MSD Trap VL Apparatus. The crystal structure was determined by Siemens $\mathrm{P}_{4}$ four circle diffractometer.

Typical one-pot procedure for the synthesis of 5a. To a $25 \mathrm{~mL}$ tube equipped with a stirring bar were added acetone (4, $2.0 \mathrm{mmol}), 2$,2-butylidene-1,3-dioxane-4,6-dione (1a, $2 \mathrm{mmol}$ ), benzaldehyde (2a, $4 \mathrm{mmol})$, aniline (3a, $1 \mathrm{mmol}$ ) and L-ascorbic acid $(0.2 \mathrm{mmol})$ in $10 \mathrm{~mL} \mathrm{CH} \mathrm{CCN}_{3}$. After the reaction was stirred vigorously for 20.0 $\mathrm{h}$ at room temperature, $\mathrm{CH}_{3} \mathrm{CN}$ was recycled by filtration. The residue was washed with water and the residue was purified by recrystallization from absolute $\mathrm{EtOH}$ and drying to afford the pure product.

1',2',4',5',7'-Pentaphenyl-1'H-dispiro[2',4',5',7',8'-tetrahydroquinoline-5,3':6',5']bis(2,2-butylidene-1,3-

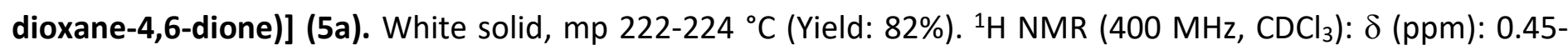
$0.58(3 \mathrm{H}, \mathrm{m}), 0.67-0.90(3 \mathrm{H}, \mathrm{m}), 1.05-1.19(2 \mathrm{H}, \mathrm{m}), 1.30-1.47(8 \mathrm{H}, \mathrm{m}), 2.56\left(1 \mathrm{H}, \mathrm{dd},{ }^{3} \mathrm{JHH}_{H} 17.6,6.0 \mathrm{~Hz}, \mathrm{H}^{\prime}-8{ }^{\prime}\right)$, 2.64-2.70 (1H, m, H''-8'), $3.99\left(1 \mathrm{H}, \mathrm{dd}, 3^{3} \mathrm{~J}_{H H} 11.6,5.6 \mathrm{~Hz}, \mathrm{H}-7^{\prime}\right), 4.62\left(1 \mathrm{H}, \mathrm{s}, \mathrm{H}-4^{\prime}\right), 4.66\left(1 \mathrm{H}, \mathrm{s}, \mathrm{H}-5^{\prime}\right), 5.23(1 \mathrm{H}, \mathrm{s}$, $\left.\mathrm{H}^{2} \mathrm{2}^{\prime}\right), 6.04\left(2 \mathrm{H}, \mathrm{t}, 3^{3} \mathrm{HH}_{\mathrm{H}} 9.2 \mathrm{~Hz}, 2 \mathrm{CH}, \mathrm{HAr}\right), 6.71\left(2 \mathrm{H}, \mathrm{dd},{ }^{3} \mathrm{~J}_{\mathrm{HH}} 14.8,7.6 \mathrm{~Hz}, 2 \mathrm{CH}, \mathrm{HAr}\right), 6.97-7.56(\mathrm{~m}, 21 \mathrm{H}, 21 \mathrm{CH}$, $\mathrm{HAr}) ;{ }^{13} \mathrm{C} \mathrm{NMR}\left(100 \mathrm{MHz}, \mathrm{CDCl}_{3}\right): \delta(\mathrm{ppm}): 22.3,22.7,22.8,32.9\left(\mathrm{C}-8{ }^{\prime}\right), 38.5,38.6,38.6,38.7,47.9$ (C-4'), 50.8(C5'), 53.0 (C-7'), 53.4, 62.0 (C-3'), 62.1(C-6'), 70.3(C-2'), $102.2\left(\mathrm{C}-4{ }^{\prime} \mathrm{a}\right), 114.1\left[(\mathrm{O})_{2} \mathrm{C}\left(\mathrm{CH}_{2}\right)_{2}\right], 114.3\left[(\mathrm{O})_{2} \mathrm{C}\left(\mathrm{CH}_{2}\right)_{2}\right]$, 126.5, 127.0, 127.2, 127.9, 128.0, 128.0, 128.2, 128.3, 128.4, 128.4, 128.7, 128.7, 129.2, 129.3, 129.5, 130.9, $131.2,131.5,135.2,136.3,137.3,138.5,142.4,144.6$ (C-8'a), 162.2(C=O), 164.4(C=O), 168.4(C=O), 169.8(C=O); $\mathrm{ESI}+\mathrm{MS} m / z=826.3[\mathrm{M}+\mathrm{H}]^{+}$.

1'-Phenyl-,2',4',5',7'-tetra(4-flurophenyl)-1'H-dispiro[2',4',5',7',8'-tetrahydroquinoline-5,3':6',5']bis(2,2-

butylidene-1,3-dioxane-4,6-dione)] (5b). White solid, mp 221-223 ${ }^{\circ} \mathrm{C}$ (Yield: $\left.\left.74 \%\right) .{ }^{1} \mathrm{H} \mathrm{NMR} \mathrm{(400} \mathrm{MHz} \mathrm{CDCl} 3\right): \delta$ (ppm): 0.59-0.70 (3H, m), 0.78-1.01 (3H, m), 1.11-1.28(2H, m), 1.38-1.49 (8H, m), 2.48-2.60 (2H, m, $\left.\mathrm{H}^{\prime}-8^{\prime}, \mathrm{H}^{\prime \prime}-8\right)$, $3.94\left(1 \mathrm{H}, \mathrm{dd}, 3^{3} \mathrm{HH}_{\mathrm{H}} 10.8,6.8 \mathrm{~Hz}, \mathrm{H}-7^{\prime}\right), 4.55$ (brs, $2 \mathrm{H}, \mathrm{H}-4^{\prime}$ and $\left.\mathrm{H}^{\prime} 5^{\prime}\right), 5.20\left(\mathrm{~s}, 1 \mathrm{H}, \mathrm{H}-2^{\prime}\right), 6.05-6.12(2 \mathrm{H}, \mathrm{m}, 2 \mathrm{CH}, \mathrm{HAr})$, 6.50-6.56 (2H, m, 2CH, HAr), 6.76-7.53 (17H, m, 17CH, HAr). $\left.{ }^{13} \mathrm{C} \mathrm{NMR} \mathrm{(100} \mathrm{MHz,} \mathrm{CDCl}\right): \delta(p p m): 22.3,22.4$, 22.8, 22.932 .9 (C-8'), 38.6, 38.8, 38.9, 39.0, 47.0 (C-4'), 49.8 (C-5'), 52.1 (C-7'), 61.9 (C-3'), 62.1 (C-6'), 69.5 (C$\left.2^{\prime}\right), 101.6$ (C-4'a), $114.4\left[(\mathrm{O})_{2} \mathrm{C}\left(\mathrm{CH}_{2}\right)_{2}\right], 114.6\left[(\mathrm{O})_{2} \mathrm{C}\left(\mathrm{CH}_{2}\right)_{2}\right], 114.8,114.9,115.0,115.0,115.1,115.1,115.2,115.3$, $115.5,115.7,126.9,128.7,130.2,130.3,130.7,130.7,130.8,130.9,131.0,131.0,131.9\left(d,{ }^{3} J_{C F} 3.1 \mathrm{~Hz}, C_{A r-F}\right)$, 132.5, $132.6\left(\mathrm{~d},{ }^{3} J_{C F} 3.1 \mathrm{~Hz}, \mathrm{C}_{\text {Ar-F}}\right), 132.7,132.9\left(\mathrm{~d},{ }^{3} J_{C F} 3.1 \mathrm{~Hz}, \mathrm{C}_{\text {Ar- } F}\right), 132.9,133.0,133.9\left(\mathrm{~d},{ }^{3} J_{C F} 3.1 \mathrm{~Hz}, \mathrm{C}_{\text {Ar-F}}\right)$, 142.6, 144.0 (C-8'a), 160.8 (d, J JF $264.5 \mathrm{~Hz}, \mathrm{C}_{\mathrm{Ar}-\mathrm{F}}$ ), 161.2 (d, J JF $264.5 \mathrm{~Hz}, \mathrm{C}_{\mathrm{Ar}-\mathrm{F}}$ ), 162.1 (C=O), 163.3 (d, J 264.5 $\left.\mathrm{Hz}, \mathrm{C}_{\mathrm{Ar}-\mathrm{F}}\right), 163.6$ (d, $\left.J_{C F} 264.5 \mathrm{~Hz}, \mathrm{C}_{\mathrm{Ar}-\mathrm{F}}\right), 164.3$ (C=O), $168.2(\mathrm{C}=\mathrm{O}), 169.6$ (C=O); ESI+MS m/z=898.3 [M+H]

1'-Phenyl-,2',4',5',7'-tetra(4-chlorophenyl)-1'H-dispiro[2',4',5',7',8'-tetrahydroquinoline-5,3':6',5']bis(2,2-

butylidene-1,3-dioxane-4,6-dione)](5c). White solid, $\mathrm{mp} 218-220{ }^{\circ} \mathrm{C}$ (Yield: $\left.77 \%\right) .{ }^{1} \mathrm{H} \mathrm{NMR}\left(400 \mathrm{MHz} \mathrm{CDCl}_{3}\right): \delta$ (ppm): 0.60-0.70 (3H, m), 0.79-1.01 (3H, m), 1.12-1.27 (2H, m), 1.40-1.49 (8H, m), $2.51\left(1 \mathrm{H}\right.$, brs, $\left.\mathrm{H}^{\prime}-8{ }^{\prime}\right), 2.53(1 \mathrm{H}$, 
brs, $\left.\mathrm{H}^{\prime \prime}-8^{\prime}\right), 3.91\left(1 \mathrm{H}, \mathrm{dd}, 3^{3} \mathrm{~J}_{H H} 10.0,7.2 \mathrm{~Hz}, \mathrm{H}-7^{\prime}\right), 4.52\left(2 \mathrm{H}, \mathrm{brs}, \mathrm{H}-4^{\prime}\right.$ and H-5'), $5.18\left(1 \mathrm{H}, \mathrm{s}, \mathrm{H}-22^{\prime}\right), 6.03-6.09(2 \mathrm{H}$, $\mathrm{m}, 2 \mathrm{CH}, \mathrm{HAr}), 6.80-6.84(2 \mathrm{H}, \mathrm{m}, 2 \mathrm{CH}, \mathrm{HAr}), 6.91-7.48(17 \mathrm{H}, \mathrm{m}, 17 \mathrm{CH}, \mathrm{HAr}) ;{ }^{13} \mathrm{C} \mathrm{NMR}(100 \mathrm{MHz}, \mathrm{CDCl}): \delta(\mathrm{ppm})$ : 22.4, 22.4, 22.8, 23.0, 32.7 (C-8'), 38.6, 38.8, 39.0, 39.1, 47.2 (C-4'), 49.8 (C-5'), 52.2 (C-7'), 61.6 (C-3'), 61.6 (C6'), 69.6 (C-2'), 101.1 (C-4'a), $114.5\left[(\mathrm{O})_{2} \mathrm{C}\left(\mathrm{CH}_{2}\right)_{2}\right], 114.7\left[(\mathrm{O})_{2} \mathrm{C}\left(\mathrm{CH}_{2}\right)_{2}\right], 127.1,128.2,128.3,128.6,128.8,128.9$, 128.9, 130.0, 130.4, 130.6, 130.8, 132.1, 132.3, 132.7, 133.2, 133.5, 133.7, 134.1, 134.4, 134.4, 135.4, 136.5, 142.8, 143.7 (C-8'a), 162.0 (C=O), 164.2 (C=O), 168.0 (C=O), 169.4 (C=O); ESI+MS m/z=962.2 [M+H] .

1'-Phenyl-2',4',5',7'-tetra(4-methylphenyl)-1'H-dispiro[2',4',5',7',8'-tetrahydro-quinoline-5,3':6',5''-bis(2,2-

butylidene-1,3-dioxane-4,6-dione)] (5d). White solid, mp $230-231{ }^{\circ} \mathrm{C}$ (Yield: $\left.65 \%\right) .{ }^{1} \mathrm{H} \mathrm{NMR}\left(400 \mathrm{MHz} \mathrm{CDCl}_{3}\right): \delta$ (ppm): 0.48-0.67 (3H, m), 0.72-0.79 (1H, m), 0.85-0.94 $(2 \mathrm{H}, \mathrm{m}), 1.09-1.26(2 \mathrm{H}, \mathrm{m}), 1.28-1.52(8 \mathrm{H}, \mathrm{m}), 2.15(3 \mathrm{H}$, $\left.\mathrm{s}, \mathrm{ArCH}_{3}\right), 2.21\left(9 \mathrm{H}, \mathrm{s}, 3 \mathrm{ArCH}_{3}\right), 2.48-2.64\left(2 \mathrm{H}, \mathrm{m}, \mathrm{H}^{\prime}-8^{\prime}, \mathrm{H}^{\prime \prime}-8^{\prime}\right), 3.92\left(1 \mathrm{H}, \mathrm{dd}, 3^{3} \mathrm{JHH}_{H} 11.6,5.6 \mathrm{~Hz}, \mathrm{H}-7^{\prime}\right), 4.54(1 \mathrm{H}, \mathrm{s}$, $\left.\mathrm{H}^{-} 4^{\prime}\right), 4.55\left(1 \mathrm{H}, \mathrm{s}, \mathrm{H}-5^{\prime}\right), 5.16\left(1 \mathrm{H}, \mathrm{s}, \mathrm{H}-2^{\prime}\right), 5.92-5.96(2 \mathrm{H}, \mathrm{m}, 2 \mathrm{CH}, \mathrm{HAr}), 6.53\left(2 \mathrm{H}, \mathrm{t}, 3^{3} \mathrm{HH} 6.8 \mathrm{~Hz}, 2 \mathrm{CH}, \mathrm{HAr}\right), 6.84-$ $7.42(17 \mathrm{H}, \mathrm{m}, 17 \mathrm{CH}, \mathrm{HAr}) ;{ }^{13} \mathrm{C} \mathrm{NMR}(100 \mathrm{MHz}, \mathrm{CDCl})$ ): $\delta(\mathrm{ppm}): 20.9\left(\mathrm{ArCH}_{3}\right), 21.0\left(\mathrm{ArCH}_{3}\right), 21.0\left(\mathrm{ArCH}_{3}\right), 21.0$ $\left(\mathrm{ArCH}_{3}\right), 22.3,22.3,22.7,22.9,33.0$ (C-8'), 38.4, 38.6, 38.6, 38.7, 47.6 (C-4'), 50.3 (C-5'), 52.6 (C-7'), $62.2\left(\mathrm{C}^{\prime} 3^{\prime}\right)$, 62.2 (C-6'), 70.1 (C-2'), 102.6 (C-4'a), 114.0 [(O) $\left.{ }_{2} \mathrm{C}\left(\mathrm{CH}_{2}\right)_{2}\right], 114.2\left[(\mathrm{O})_{2} \mathrm{C}\left(\mathrm{CH}_{2}\right)_{2}\right], 126.3,128.3,128.4,128.5,128.7$, 129.0, 129.0, 129.2, 129.3, 130.8, 131.1, 131.4, 132.1, 133.2, 134.2, 135.7, 136.5, 136.7, 137.5, 137.9, 142.8, 144.6 (C-8'a), 162.3 (C=O), 164.6 (C=O), 168.6 (C=O), 170.0 (C=O); ESI+MS m/z=882.4 [M+H] .

1'-Phenyl-2',4',5',7'-tetra(4-methoxylphenyl)-1'H-dispiro[2',4',5',7',8'-tetrahydro-quinoline-5,3':6',5''-bis(2,2butylidene-1,3-dioxane-4,6-dione)] (5e). Yellow solid, mp 196-198 ${ }^{\circ} \mathrm{C}$ (Yield: 63\%). $\left.{ }^{1} \mathrm{H} \mathrm{NMR} \mathrm{(400} \mathrm{MHz} \mathrm{CDCl}_{3}\right)$ : $\delta$ (ppm): 0.57-0.73 (3H, m), 0.78-1.01 (3H, m), 1.12-1.46 (10H, m), 2.46-2.61 (2H, m, H'-8', H'-8'), $3.66(3 \mathrm{H}, \mathrm{s}$, $\left.\mathrm{ArCH}_{3} \mathrm{O}\right), 3.70\left(3 \mathrm{H}, \mathrm{s}, \mathrm{ArCH}_{3} \mathrm{O}\right), 3.72\left(6 \mathrm{H}, \mathrm{s}, 2 \mathrm{ArCH}_{3} \mathrm{O}\right), 3.92\left(1 \mathrm{H}, \mathrm{dd},{ }^{3}{ }^{\mathrm{HH}} 12.0,6.0 \mathrm{~Hz}, \mathrm{H}-7{ }^{\prime}\right), 4.51(2 \mathrm{H}, \mathrm{brs}, \mathrm{H}-4$ ', $\left.\mathrm{H}-5^{\prime}\right), 5.14\left(1 \mathrm{H}, \mathrm{s}, \mathrm{H}-2^{\prime}\right), 5.98-6.03(2 \mathrm{H}, \mathrm{m}, 2 \mathrm{CH}, \mathrm{HAr}), 6.29-6.34(2 \mathrm{H}, \mathrm{m}, 2 \mathrm{CH}, \mathrm{HAr}), 6.57-7.46(17 \mathrm{H}, \mathrm{m}, 17 \mathrm{CH}$, $\mathrm{HAr}) ;{ }^{13} \mathrm{C} \mathrm{NMR}\left(100 \mathrm{MHz}, \mathrm{CDCl}_{3}\right): \delta(\mathrm{ppm}): 22.3,22.3,22.8,22.9,33.1$ (C-8'), 38.5, 38.7, 38.8, 38.9, 47.1 (C-4'), $\left.49.8\left(\mathrm{C}^{-} 5^{\prime}\right), 52.1\left(\mathrm{C}-7^{\prime}\right), 55.1\left(\mathrm{CH}_{3} \mathrm{O}\right), 55.2\left(\mathrm{CH}_{3} \mathrm{O}\right), 55.2\left(\mathrm{CH}_{3} \mathrm{O}\right), 55.4\left(\mathrm{CH}_{3} \mathrm{O}\right), 62.2(\mathrm{C}-3)^{\prime}\right), 62.3\left(\mathrm{C}-6^{\prime}\right), 69.9\left(\mathrm{C}^{\prime} 2^{\prime}\right)$, 102.8 (C-4'a), $112.6\left[(\mathrm{O})_{2} \mathrm{C}\left(\mathrm{CH}_{2}\right)_{2}\right], 113.5,113.6,113.7,114.0,114.1,114.1,114.2,126.3,127.2,128.4,128.4$, 129.5, 129.7, 130.2, 130.4, 130.7, 132.1, 132.1, 132.5, 142.2, 144.6 (C-8'a), 158.7 (C $\left.\mathrm{C}_{\text {Ar-o }}\right), 158.8$ (C $\left.\mathrm{C}_{\mathrm{Ar}-\mathrm{O}}\right), 159.1$ $\left(C_{\text {Ar-O }}\right), 159.3$ ( $\left.C_{\text {Ar-O }}\right), 162.4(C=O), 164.6(C=0), 168.7(C=O), 170.2(C=O) ; E S I+M S m / z=946.4[M+H]^{+}$.

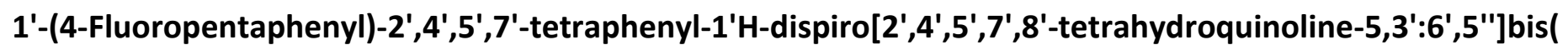
2,2-butylidene-1,3-dioxane-4,6-dione)] (5f). White solid, mp 224-227 ${ }^{\circ} \mathrm{C}$ (Yield: $\left.72 \%\right) .{ }^{1} \mathrm{H} \mathrm{NMR} \mathrm{(400} \mathrm{MHz,}$ $\left.\mathrm{CDCl}_{3}\right): \delta(\mathrm{ppm}): 0.45-0.58(3 \mathrm{H}, \mathrm{m}), 0.67-0.74(1 \mathrm{H}, \mathrm{m}), 0.82-.90(2 \mathrm{H}, \mathrm{m}), 1.06-1.20(2 \mathrm{H}, \mathrm{m}), 1.23-1.46(8 \mathrm{H}, \mathrm{m})$, $2.52\left(1 \mathrm{H}, \mathrm{dd},{ }^{3} \mathrm{~J}_{H H} 17.2,5.6 \mathrm{~Hz}, \mathrm{H}^{\prime}-8^{\prime}\right), 2.60-2.69\left(1 \mathrm{H}, \mathrm{m}, \mathrm{H}^{\prime \prime}-8^{\prime}\right), 3.98\left(1 \mathrm{H}, \mathrm{dd},{ }^{3} \mathrm{~J}_{H H} 12.0,6.4 \mathrm{~Hz}, \mathrm{H}^{\prime}-7^{\prime}\right), 4.61(1 \mathrm{H}, \mathrm{s}$, $\left.\mathrm{H}^{-4} 4^{\prime}\right), 4.64\left(1 \mathrm{H}, \mathrm{s}, \mathrm{H}-5^{\prime}\right), 5.16\left(1 \mathrm{H}, \mathrm{s}, \mathrm{H}-2^{\prime}\right), 6.04\left(2 \mathrm{H}, \mathrm{t},{ }^{3} \mathrm{~J}_{H H} 8.4 \mathrm{~Hz}, 2 \mathrm{CH}, \mathrm{HAr}\right), 6.72\left(2 \mathrm{H}, \mathrm{ddd}, 3^{3} \mathrm{HH}^{15}, 6 \mathrm{~Hz},{ }^{4} J_{H H}\right.$

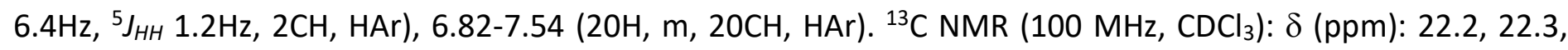
22.7, 22.8, 33.0 (C-8'), 38.5, 38.5, 38.6, 38.8, 47.8 (C-4'), 50.8 (C-5'), $53.0\left(\mathrm{C}^{\prime} 7^{\prime}\right), 61.8\left(\mathrm{C}-3^{\prime}\right), 62.0$ (C-6'), $70.4(\mathrm{C}-$ $\left.2^{\prime}\right), 102.8$ (C-4'a), $114.2\left[(\mathrm{O})_{2} \mathrm{C}\left(\mathrm{CH}_{2}\right)_{2}\right], 114.4\left[(\mathrm{O})_{2} \mathrm{C}\left(\mathrm{CH}_{2}\right)_{2}\right], 127.1,127.2,128.0,128.0,128.0,128.1,128.4$, $128.5,128.5,128.6,128.7,129.0,129.3,129.4,130.9,131.2,131.5,135.0,136.1,137.1,138.4,140.7\left(d,{ }^{2} J_{C F} 3.2\right.$ $\left.\mathrm{Hz}, \mathrm{C}_{\mathrm{Ar}}\right), 142.3,143.8$ (C-8'a), 160.8 (d, $\left.{ }^{1} \mathrm{~J}_{\mathrm{CF}} 244.6 \mathrm{~Hz}, \mathrm{C}_{\mathrm{Ar}}\right), 162.2(\mathrm{C}=0), 164.5(\mathrm{C}=0), 168.3$ (C=O), 169.7 (C=O); $\mathrm{ESI}+\mathrm{MS} m / z=844.3[\mathrm{M}+\mathrm{H}]^{+}$.

1'-(4-Methoxylphenyl)-2',4',5',7'-tetraphenyl-1'H-dispiro[2',4',5',7',8'-tetrahydro-quinoline-5,3':6',5"'bis(2,2-butylidene-1,3-dioxane-4,6-dione)] (5g). White solid, mp 202-204 ${ }^{\circ} \mathrm{C}$ (Yield: 70\%). ${ }^{1} \mathrm{H} \mathrm{NMR}(400 \mathrm{MHz}$, $\left.\mathrm{CDCl}_{3}\right): \delta$ (ppm): 0.44-0.58 (3H, m), 0.67-0.74 (1H, m), 0.82-0.89 (2H, m), 1.06-1.18 (2H, m), 1.26-1.45 (8H, m), $2.53\left(1 \mathrm{H}, \mathrm{dd},{ }^{3} \mathrm{~J}_{H H} 17.2,5.2 \mathrm{~Hz}, \mathrm{H}^{\prime}-8^{\prime}\right), 2.62-2.70\left(1 \mathrm{H}, \mathrm{m}, \mathrm{H}^{\prime \prime}-8 \mathrm{\prime}\right), 3.67\left(3 \mathrm{H}, \mathrm{s}, \mathrm{ArOCH}_{3}\right), 3.98\left(1 \mathrm{H}, \mathrm{dd},{ }^{3} \mathrm{~J}_{H H} 12.0,5.2\right.$ Hz, H-7'), $4.60\left(1 \mathrm{H}, \mathrm{s}, \mathrm{H}-4^{\prime}\right), 4.64\left(1 \mathrm{H}, \mathrm{s}, \mathrm{H}^{\prime} 5^{\prime}\right), 5.17$ (1H, s, H-2'), 6.01-6.06 (2H, m, 2CH, HAr), 6.68-6.74 (2H, m, $2 \mathrm{CH}, \mathrm{HAr}), 6.96-7.55(2 \mathrm{OH}, \mathrm{m}, 20 \mathrm{CH}, \mathrm{HAr}) ;{ }^{13} \mathrm{C} \mathrm{NMR}\left(100 \mathrm{MHz}, \mathrm{CDCl}_{3}\right): \delta(\mathrm{ppm}): 22.2,22.2,22.7,22.8,32.9(\mathrm{C}-$ $\left.8^{\prime}\right), 38.5,38.6,38.6,38.7,47.9\left(C^{\prime}-4^{\prime}\right), 50.7\left(C^{\prime}-5^{\prime}\right), 53.1\left(C^{\prime}-7^{\prime}\right), 55.1\left(\mathrm{OCH}_{3}\right), 62.0\left(\mathrm{C}-3^{\prime}\right), 62.1\left(\mathrm{C}-6^{\prime}\right), 70.4\left(\mathrm{C}-2^{\prime}\right)$, 
101.9 (C-4'a), $114.1\left[(\mathrm{O})_{2} \mathrm{C}\left(\mathrm{CH}_{2}\right)_{2}\right], 114.3\left[(\mathrm{O})_{2} \mathrm{C}\left(\mathrm{CH}_{2}\right)_{2}\right], 127.0,127.9,127.9,127.9,128.0,128.3,128.3,128.4$, 128.4, 128.7, 128.7, 129.1, 129.3, 129.5, 130.8, 131.2, 131.5, 135.3, 136.3, 137.3, 138.6, 142.9 (C-8'a), 157.6 ( $\left.\mathrm{C}_{\text {Ar-O }}\right), 162.2(\mathrm{C}=0), 164.5(\mathrm{C}=0), 168.4(\mathrm{C}=0), 169.8(\mathrm{C}=0)$; $\mathrm{ESI}+\mathrm{MS} m / z=856.3[\mathrm{M}+\mathrm{H}]^{+}$.

1'-(4-Methylphenyl)-2',4',5',7'-tetraphenyl-1'H-dispiro[2',4',5',7',8'-tetrahydro-quinoline-5,3':6',5'"-bis(2,2butylidene-1,3-dioxane-4,6-dione)] (5h). White solid, mp 221-223 ${ }^{\circ} \mathrm{C}$ (Yield: 76\%). $\left.{ }^{1} \mathrm{H} \mathrm{NMR} \mathrm{(400} \mathrm{MHz,} \mathrm{CDCl}_{3}\right): \delta$ (ppm): 0.44-0.58 (3H, m), 0.67-0.74 (1H, m), 0.81-0.88 (2H, m), 1.05-1.18 (2H, m), 1.26-1.43 (8H, m), $2.17(3 \mathrm{H}$, $\left.\mathrm{s}, \mathrm{ArCH}_{3}\right), 2.54\left(1 \mathrm{H}, \mathrm{dd}, 3^{3} \mathrm{~J}_{H H} 17.2,5.6 \mathrm{~Hz}, \mathrm{H}^{\prime}-8^{\prime}\right), 2.61-2.70\left(1 \mathrm{H}, \mathrm{m}, \mathrm{H}^{\prime \prime}-8 \mathrm{\prime}\right), 3.98\left(1 \mathrm{H}, \mathrm{dd}, 3^{3} \mathrm{JH}_{12} 12.0,5.6 \mathrm{~Hz}, \mathrm{H}^{\prime} \mathbf{7}^{\prime}\right)$, $4.61\left(1 \mathrm{H}, \mathrm{s}, \mathrm{H}-4^{\prime}\right), 4.64\left(1 \mathrm{H}, \mathrm{s}, \mathrm{H}^{-} 5^{\prime}\right), 5.20\left(1 \mathrm{H}, \mathrm{s}, \mathrm{H}-2^{\prime}\right), 6.01-6.06(1 \mathrm{H}, \mathrm{m}, 2 \mathrm{CH}, \mathrm{HAr}), 6.68-6.74(1 \mathrm{H}, \mathrm{m}, 2 \mathrm{CH}, \mathrm{HAr})$, 6.92-7.55 (20H, m, 20CH, HAr); ${ }^{13} \mathrm{C}$ NMR (100 MHz, $\left.\mathrm{CDCl}_{3}\right): \delta(\mathrm{ppm}): 21.0,21.0,22.2,22.7,22.8,32.8$ (C-8'), 38.4, 38.5, 38.6, 38.6, 47.9 (C-4'), 50.7 (C-5'), 53.0 (C-7'), 62.0 (C-3'), 62.1 (C-6'), 70.2 (C-2'), 101.9 (C-4'a), 114.1 $\left[(\mathrm{O})_{2} \mathrm{C}\left(\mathrm{CH}_{2}\right)_{2}\right], 114.3\left[(\mathrm{O})_{2} \mathrm{C}\left(\mathrm{CH}_{2}\right)_{2}\right], 127.0,127.1,127.9,127.9,128.0,128.2,128.3,128.4,128.4,128.6,128.7$, $129.2,129.3,129.5,130.8,131.2,131.5,135.3,136.0,136.3,137.3,138.6,141.8,142.7$ (C-8'a), 162.2 (C=O), $164.4(\mathrm{C}=\mathrm{O}), 168.6(\mathrm{C}=\mathrm{O}), 169.8(\mathrm{C}=\mathrm{O}) ; \mathrm{ESI}+\mathrm{MS} \mathrm{m} / \mathrm{z}=840.4[\mathrm{M}+\mathrm{H}]^{+}$.

1',2',4',5',7'-Pentaphenyl-1'H-dispiro[2',4',5',7',8'-tetrahydroquinoline-5,3':6',5']bis(2,2-pentylidene-1,3dioxane-4,6-dione)](5i). White solid, $\mathrm{mp} 220-221{ }^{\circ} \mathrm{C}$ (Yield: $\left.82 \%\right) .{ }^{1} \mathrm{H} \mathrm{NMR}\left(400 \mathrm{MHz}, \mathrm{CDCl}_{3}\right): \delta$ (ppm): $0.09-$ $0.35(4 \mathrm{H}, \mathrm{m}), 0.55-0.71(4 \mathrm{H}, \mathrm{m}), 0.96-1.28(12 \mathrm{H}, \mathrm{m}), 2.56\left(1 \mathrm{H}, \mathrm{dd},{ }^{3} J_{H H} 17.2,5.6 \mathrm{~Hz}, \mathrm{H}^{\prime}-8{ }^{\prime}\right), 2.61-2.69\left(1 \mathrm{H}, \mathrm{m}, \mathrm{H}^{\prime \prime}-\right.$ $\left.8^{\prime}\right), 4.00\left(1 \mathrm{H}, \mathrm{dd},{ }^{3} J_{H H} 11.6,5.6 \mathrm{~Hz}, \mathrm{H}-7^{\prime}\right), 4.63\left(1 \mathrm{H}, \mathrm{s}, \mathrm{H}-4^{\prime}\right), 4.66\left(1 \mathrm{H}, \mathrm{s}, \mathrm{H}-5^{\prime}\right), 5.23\left(1 \mathrm{H}, \mathrm{s}, \mathrm{H}-2^{\prime}\right), 6.04\left(2 \mathrm{H}, \mathrm{t},{ }^{3} \mathrm{~J}_{H H}\right.$

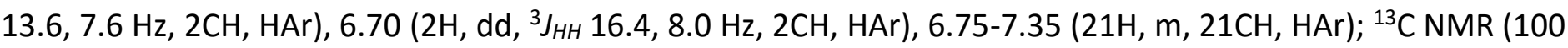
$\mathrm{MHz} \mathrm{CDCl}_{3}$ ): $\delta$ (ppm): 21.6, 21.8, 21.8, 23.7, 32.8 (C-8'), 36.5, 36.9, 37.7, 37.7, 47.8 (C-4'), 50.8 (C-5'), 53.1 (C$\left.7^{\prime}\right), 62.1$ (C-3'), 62.3 (C-6'), 70.3 (C-2'), 102.3 (C-4'a), 105.7 [(O) $\left.{ }_{2} \mathrm{C}\left(\mathrm{CH}_{2}\right)_{2}\right], 105.9$ [(O) $\left.{ }_{2} \mathrm{C}\left(\mathrm{CH}_{2}\right)_{2}\right], 126.5,127.0$, $127.1,127.8,127.8,127.9,128.0,128.3,128.3,128.3,128.4,128.6,128.8,129.4,129.5,130.82,131.4,131.6$, 135.3, 136.5, 137.4, 138.5, 142.3, 144.8 (C-8'a), 162.1 (C=0), 164.4 (C=0), 168.4 (C=0), 169.7 (C=O); ESI+MS $\mathrm{m} / \mathrm{z}=854.4[\mathrm{M}+\mathrm{H}]^{+}$.

1',2',4',5',7'-Pentaphenyl-1'H-dispiro[2',4',5',7',8'-tetrahydroquinoline-5,3':6',5'']bis(2,2-methyl-1,3-dioxane4,6-dione)] (5j). White solid, $\mathrm{mp} 246-248{ }^{\circ} \mathrm{C}$ (Yield: $\left.85 \%\right) .{ }^{1} \mathrm{H} \mathrm{NMR}\left(400 \mathrm{MHz}, \mathrm{CDCl}_{3}\right): \delta$ (ppm): $0.35\left(3 \mathrm{H}, \mathrm{s}, \mathrm{CH}_{3}\right)$, $0.37\left(3 \mathrm{H}, \mathrm{s}, \mathrm{CH}_{3}\right), 0.59\left(3 \mathrm{H}, \mathrm{s}, \mathrm{CH}_{3}\right), 0.61\left(3 \mathrm{H}, \mathrm{s}, \mathrm{CH}_{3}\right), 2.56\left(1 \mathrm{H}, \mathrm{dd},{ }^{3} \mathrm{~J}_{H H} 17.6,6.0 \mathrm{~Hz}, \mathrm{H}^{\prime}-8^{\prime}\right), 2.61-2.69\left(1 \mathrm{H}, \mathrm{m}, \mathrm{H}^{\prime \prime}-\right.$ $\left.8^{\prime}\right), 4.02\left(1 \mathrm{H}, \mathrm{dd}, 3^{3} \mathrm{JHH}_{12} 12.0,6.0 \mathrm{~Hz}, \mathrm{H}_{-1} \mathrm{7}^{\prime}\right), 4.64\left(1 \mathrm{H}, \mathrm{s}, \mathrm{H}-4^{\prime}\right), 4.66\left(1 \mathrm{H}, \mathrm{s}, \mathrm{H}-5^{\prime}\right), 5.25\left(1 \mathrm{H}, \mathrm{s}, \mathrm{H}-2^{\prime}\right), 6.03\left(1 \mathrm{H}, \mathrm{d}, 3^{3} \mathrm{~J}_{H H}\right.$ $7.6 \mathrm{~Hz}, 1 \mathrm{CH}, \mathrm{HAr}), 6.06\left(1 \mathrm{H}, \mathrm{d},{ }^{3} J_{H H} 7.6 \mathrm{~Hz}, 1 \mathrm{CH}, \mathrm{HAr}\right), 6.69\left(1 \mathrm{H}, \mathrm{d},{ }^{3} J_{H H} 7.6 \mathrm{~Hz}, 1 \mathrm{CH}, \mathrm{HAr}\right), 6.73\left(1 \mathrm{H}, \mathrm{d},{ }^{3} J_{H H} 8.0 \mathrm{~Hz}\right.$, $1 \mathrm{H}, 1 \mathrm{CH}, \mathrm{HAr}), 6.98-7.57(21 \mathrm{H}, \mathrm{m}, 21 \mathrm{CH}, \mathrm{HAr}) .{ }^{13} \mathrm{C} \mathrm{NMR}\left(100 \mathrm{MHz}, \mathrm{CDCl}_{3}\right): \delta(\mathrm{ppm}): 28.0\left(\mathrm{CH}_{3}\right), 28.2\left(\mathrm{CH}_{3}\right), 28.5$ $\left(\mathrm{CH}_{3}\right), 28.6\left(\mathrm{CH}_{3}\right), 32.7\left(\mathrm{C}-8^{\prime}\right), 47.6\left(\mathrm{C}^{\prime} 4^{\prime}\right), 50.8$ (C-5'), 53.1 (C-7'), $61.6\left(\mathrm{C}-3^{\prime}\right), 61.8\left(\mathrm{C}-6^{\prime}\right), 70.1\left(\mathrm{C}-2{ }^{\prime}\right), 102.1\left(\mathrm{C}-4^{\prime} \mathrm{a}\right)$, $105.3\left[\mathrm{C}\left(\mathrm{CH}_{3}\right)_{2}\right], 105.5\left[\mathrm{C}\left(\mathrm{CH}_{3}\right)_{2}\right], 126.6,127.1,127.2,127.9,128.0,128.0,128.1,128.4,128.4,128.5,128.5$, $128.7,128.8,129.4,129.4,129.5,130.8,131.3,131.6,135.2,136.2,137.2,138.5,142.3,144.6$ (C-8'a), 161.9 $(\mathrm{C}=0), 164.1(\mathrm{C}=0), 168.2(\mathrm{C}=\mathrm{O}), 169.5(\mathrm{C}=0)$; $\mathrm{ESI}+\mathrm{MS} \mathrm{m} / \mathrm{z}=774.3[\mathrm{M}+\mathrm{H}]^{+}$.

X-ray diffraction experiment: the X-ray diffraction measurements were made onSiemens $\mathrm{P}_{4}$ four circle diffractometer, using $\mathrm{CuK}_{\mathrm{a}}(\lambda=1.54180 \AA)$. A colourless prismatic crystal of the title compound, of dimensions $0.18 \times 0.16 \times 0.12 \mathrm{~mm}^{3}$, was mounted on a glass fibre and used for data collection.

\section{Acknowledgements}

This work was supported by the Science and technology research project of Jiangxi Provincial Education Department (No.GJJ170170, GJJ180892). We also thank the support from Jiangxi Normal University doctoral research initiation Fund Project (No.JXSDBY2020-B012). 


\section{References}

1. Michael, J. P. Nat. Prod. Rep. 1997, 14, 605.

https://doi.org/10.1039/np9971400605

2. Mohamed, E. A. Chem.Pap. 1994, 48, 261. https://doi.org/10.1016/0166-218X(92)00179-P

3. Cai, S. X.; Zhou, Z. L.; Huang, J. C.; Whittermore, E. R.; Egbuwoku, Z. O.; Lü, Y. X.; Hawkinson, J. E. Woodward, R. M.; Weber, E.; Keana, J. F. W. J. Med. Chem. 1996, 39, 3248.

4. Asolkar, R. N.; Schroeder, D.; Heckmann, R.; Lang, S.; Wagner-Doebler, I.; Laatsch, H. J. Antibiot. 2004, 57, 17. https://doi.org/10.7164/antibiotics.57.17

5. Alonso, C.; Fuertes, M.; Martin-Encinas, E.; Selas, A.; Rubiales, G.; Tesauro, C.; Knudssen, B. K.; Palacios, F. Eur. J. Med. Chem. 2018, 149, 225.

https://doi.org/10.1002/ejic.201701462

6. Gholap, A. R.; Toti, K. S.; Shirazi, F.; Kumari, R.; Bhat, M. K.; Deshpande, M. V.; Srinivasan, K. V. Bioorg. Med. Chem. 2007, 15, 6705.

7. Fabio, R. D.; Alvaro, G.; Bertani, B.; Donati, D.; Pizzi, D. M.; Gentile, G.; Pentassuglia, G.; Giacobbe, S.; Spada, S.; Ratti, E.; Corsi, M.; Quartaroli, M.; Barnaby, R. J.; Vitulli, G. Bioorg. Med. Chem. Lett. 2007, 17, 1176.

https://doi.org/10.1016/j.bmcl.2006.12.022

8. Ghorab, M. M.; Ragab, F. A.; Hamed, M. M. Eur. J. Med. Chem. 2009, 44, 4211. https://doi.org/10.1016/j.ejmech.2009.05.017

9. Güiza, F. M.; Duarte, Y. B.; Mendez-Sanchez, S. C.; Bohórquez, A. R. R. Med. Chem. Res. 2019, $28,1182$. https://doi.org/10.1007/s00044-019-02363-z

10. Kumar, A.; Srivastava, S.; Gupta, G.; Chaturvedi, V.; Sinha, S.; Srivastava, R. ACS Comb. Sci. 2011, 13, 65. https://doi.org/10.1021/co100022h

11. Ramnauth, J.; Speed, J.; Maddaford, S. P.; Dove, P.; Annedi, S. C.; Renton, P.; Rakhit, S.; Andrew, J.; Silverman, S.; Mladenova, G.; Zinghini, S.; Nair, S.; Catalano, C.; Lee, D. K. H. Felice, D. M.; Porreca, F. J. Med. Chem. 2011, 54, 5562.

12. Schrader, T. O.; Kasem, M.; Ren, A.; Feichtinger, K.; Doori, B. A. Wei, J.; Wu, C. R.; Dang, H.; Le, M.; Gatlin, J.; Chase, K.; Dong, J.; Whelan, K. T. Sage, C.; Grottick, A. J.; Semple, G. Bioorg. Med. Chem. Lett. 2016, 26, 5877. https://doi.org/10.1016/j.bmcl.2016.11.016

13. Calhoun, W.; Carlson, R. P.; Crossley, R.; Datko, L. J.; Dietrich, S.; Heatherington, K.; Marshall, L. A.; Meade, P. J.; Opalko, A.; Shepherd, R. G. J. Med. Chem. 1995, 38, 1473.

https://doi.org/10.1021/jm00009a008

14. Gudmundsson, K. S.; Boggs, S. D.; Catalano, J. G.; Svolto, A.; Spaltenstein, A.; Thomson, M.; Wheelan, P.; Jenkinson, S. Bioorg. Med. Chem. Lett. 2009, 19, 6399.

https://doi.org/10.1016/i.bmcl.2009.09.056

15. Chande, M. S.; Khanwelkar, R. R. Tetrahedron Lett. 2005, 46, 7787.

https://doi.org/10.1016/i.tetlet.2005.09.030

16. Ivanov, A. S. Chem. Soc. Rev. 2008, 37, 789.

https://doi.org/10.1039/b716020h

17. Fan, N. L.; Xu, Z. H.; Xiang, Z. B.; Xiao, Q.; Liao, C. W. Chin. J. Org. Chem. 2019, 39, 2892. 
18. Xu, Z. H.; Zhou, C. L.; Xiang, Z. B.; Xiao, Q. Arkivoc 2019, (vi), 158. https://doi.org/10.24820/ark.5550190.p011.046

19. Lashkari, M.; Maghsoodlou, M. T.; Hazeri, N.; Habibi-Khorassani, S. M.; Akbarzadeh-Torbati, N.; GarcíaGranda, S.; Torre-Fernández, L. J. Heterocycl. Chem. 2015, 52, 873.

20. Salahi, S.; Hazeri, N.; Maghsoodlou, M. T.; García-Granda, S.; Torre- Fernández, L. J. Chem. Res. 2014, 38, 383.

21. Hazeri, N.; Lashkari, M.; García-Granda, S.; Torre-Fernández, L. Aust. J. Chem. 2014, 67, 1656. https://doi.org/10.1071/CH13713

22. Salahi, S.; Maghsoodlou, M. T.; Hazeri, N.; Lashkari, M.; Garcia-Granda, S.; Torre-Fernandez, L. Chin. J. Catal. 2015, 36, 1023. https://doi.org/10.3109/01612840.2015.1128257

23. Salahi, S.; Hazeri, N.; Maghsoodlou, M. T.; Lashkari, M.; Torbati, N. A.; Garcia-Granda, S.; Torre-Fernandez, L. J. Chil. Chem. Soc. 2018, 63, 4159. https://doi.org/10.4067/S0717-97072018000404159

24. Fatahpour, M.; Lashkari, M.; Hazeri, N.;Sadeh, F. N.; Maghsoodlou, M. T. Org. Prep. Proced. Int. 2019, 51, 576. https://doi.org/10.1080/00304948.2019.1677992

25. Salahi, S.; Maghsoodlou, M. T.; Hazeri, N.; Lashkari, M.; Torbati, N. A.; Kazemian, M. A. García-Granda, S.; Torre-Fernández, L. J. Saudi Chem. Soc. 2016, 20, 349.

https://doi.org/10.1016/j.jscs.2014.11.002

26. Xu, Z. H.; Chen, F. B.; Li, Y. Y.; Huang, Q. S.; Liao, C. W. Chin. J. Org. Chem. 2018, 38, 3101.

27. Pariyar, G. C.; Mitra, B.; Mukherjee, S.; Ghosh, P. ChemistrySelect 2020, 5, 104. https://doi.org/10.1002/slct.202002629

28. Stebbinsa, N. B.; Howarda, L. R.; Priora, R. L.; Brownmillera, C.; Liyanageb, R.; Layb, J. O.; Yang, X. Y.; Qianc, S. Y. J. Berry Res. 2016, 6, 175.

29. Lawrence, G. D.; Yuan, D. M. J. Agric. Food Chem. 1996, 44, 3461. https://doi.org/10.1021/jf960079k

30. Das, S. K.; Bhattacharjee, P.; Bora, U. ChemistrySelect 2018, 3, 2131. https://doi.org/10.1002/slct.201703036

31. Xu, Z. H.; Li, Y. J.; Liu, D. Y.; Xiao, Q. Chin. J. Org. Chem. 2018, 38, 3118. https://doi.org/10.6023/cjoc201804005

32. Zhou, P.; Xu, Z. H. Chin. J. Jiangxi Normal Uni.(Natl. Sci.) 2019, 43, 167.

33. Yan, N.; Xiong, B.; Liao, W. L.; Xu, Z. H. Chin. J. Org. Chem. 2010, 30, 1391. 